\title{
Diffuse and targeted searches for ultra-high-energy photons using the hybrid detector of the Pierre Auger Observatory
}

\author{
Marcus Niechciol ${ }^{* a}$ for the Pierre Auger Collaboration ${ }^{b}$ \\ ${ }^{a}$ Department Physik, Universität Siegen, 57068 Siegen, Germany \\ ${ }^{b}$ Observatorio Pierre Auger, Av. San Martín Norte 304, 5613 Malargüe, Argentina \\ E-mail: auger_spokespersons@fnal.gov \\ Full author list: http://www.auger.org/archive/authors_icrc_2017.html
}

\begin{abstract}
Two hybrid analyses using air shower events recorded by both the Surface Detector Array (SD) and the Fluorescence Detector (FD) of the Pierre Auger Observatory are presented. In the first analysis, a search for a diffuse flux of photons with energies above $10^{18} \mathrm{eV}=1 \mathrm{EeV}$ is performed. An unprecedented separation power between photon and hadron primaries is achieved through combining observables from both the FD and the SD. The upper limits on the photon flux determined in this analysis are the most stringent limits to date at these energies, reaching the expected flux of cosmogenic photons in some astrophysical scenarios and placing severe constraints on non-standard models for the production of UHE cosmic rays.

In the second analysis, a targeted search for $\mathrm{EeV}$ photon point sources is performed using several classes of galactic and extragalactic candidate objects. No significant excess of photon-like air shower events is found for any of the source candidates. The photon flux limits for selected source candidates constrain theoretical models for $\mathrm{EeV}$ proton production at non-transient Galactic and nearby extragalactic sources, and in particular at the Galactic center region.
\end{abstract}

35th International Cosmic Ray Conference - ICRC2017

10-20 July, 2017

Bexco, Busan, Korea

${ }^{*}$ Speaker. 


\section{Introduction}

Photons are the main messenger particles for exploring the Universe. They are observed in a broad energy range, spanning from the radio regime, through the visible light up to the X-ray and gamma-ray regimes. The maximum photon energy that has been observed so far is in the order of $100 \mathrm{TeV}$, detected using ground-based air Cherenkov telescopes [1]. At ultra-high energies, in the $\mathrm{EeV}$ regime, only charged cosmic rays have been detected so far. However, the nature and origin of these ultra-high-energy (UHE) cosmic rays is not yet known. A discovery of UHE photons could help to answer fundamental questions about UHE cosmic rays. For example, UHE photons are tracers of the Greisen-Zatsepin-Kuzmin (GZK) process, i.e. the interaction of UHE protons with photons from the cosmic microwave background (CMB). In these interactions, neutral pions are produced, which subsequently decay into pairs of UHE photons. If these predicted GZK photons were observed, it would be an indicator for the GZK process being the reason for the observed suppression in the energy spectrum of UHE cosmic rays [2].

Due to their small incoming flux (less than one particle per square kilometer per year), UHE cosmic particles impinging on the Earth can only be detected indirectly by measuring the extensive air showers they initiate when entering the Earth's atmosphere. For the identification of primary photons in the recorded air shower data, the differences between air showers initiated by primary photons and those induced by primary hadrons are of great importance [3]. On average, air showers initiated by UHE photons develop deeper in the atmosphere than showers of the same primary energy induced by hadrons, due to the typically smaller multiplicity of electromagnetic interactions. This can be expressed through the observable $X_{\max }$, which describes the atmospheric depth of the shower maximum. Another key difference is the lower average number of muons in photon-induced air showers compared to air showers intiated by hadrons, a consequence of the smaller cross section for photo-nuclear interactions compared to the cross sections for electromagnetic interactions.

The Pierre Auger Observatory [4], located near Malargüe, Argentina, offers an unprecedented exposure for UHE cosmic particles. A key feature of the Pierre Auger Observatory is the hybrid concept, combining a Surface Detector Array (SD) with a Fluorescence Detector (FD). The SD consists of 1660 water-Cherenkov detectors arranged on a triangular grid with a spacing of $1.5 \mathrm{~km}$, covering a total area of more than $3000 \mathrm{~km}^{2}$. The SD is overlooked by 27 fluorescence telescopes, located at four sites at the border of the array. The SD samples the lateral shower profile at ground level, i.e. the distribution of particles as a function of the distance from the shower axis, with a duty cycle of $\sim 100 \%$, while the FD records the longitudinal shower development in the atmosphere above the SD. The FD can only be operated in clear, moonless nights, reducing the duty cycle to $\sim 13 \%$. Combining measurements from both detector systems in hybrid events yields data of superior quality, which are exploited in the two analyses presented in the following sections. In the first analysis, a search for a diffuse flux of photons with energies above $10^{18} \mathrm{eV}$ is performed [5], while the second analysis is a targeted search for point sources of $\mathrm{EeV}$ photons [6].

\section{Search for a diffuse flux of photons}

The main observable for the search for photons with hybrid data is $X_{\max }$, since it can be directly measured with the FD. To make full use of the information available in hybrid events, $X_{\max }$ is com- 
plemented with observables related to the SD measurement of the air shower. The lateral shower profile, can be described by a Lateral Distribution Function (LDF). In general, photon-induced air showers have a steeper LDF and consequently a smaller footprint on ground (and thus a smaller number of triggered SD stations $N_{\text {stat }}$ ) compared to those initiated by hadrons. The differences in the LDF between the different primary particle types can be exploited with the observable $S_{b}$ [7]:

$$
S_{b}=\sum_{i}^{N_{\text {stat }}} S_{i}\left(\frac{r_{i}}{r_{0}}\right)^{b},
$$

where $S_{i}$ and $r_{i}$ are the signal, measured in units of Vertical Equivalent Muon (VEM), and the distance from the shower axis of the $i$-th station, $r_{0}=1000 \mathrm{~m}$ is a reference distance and $b$ is a constant [5].

The photon/hadron discrimination has been studied using detailed MC simulations of the air showers and the detector response with primary energies between $10^{17}$ and $10^{20} \mathrm{eV}$ and zenith angles between 0 and $65^{\circ}$. In Fig. 1, the correlation between the three discriminating observables $X_{\max }, S_{b}$ and $N_{\text {stat }}$ is shown exemplarily for the energy range between $10^{18}$ and $10^{19} \mathrm{eV}$. To fully extract the separation power of the discriminating observables, a multivariate analysis (MVA) is performed. Different algorithms and combinations of input observables have been tested (see Fig. 2, left). A boosted decision tree (BDT) combining $X_{\max }, S_{b}$ and $N_{\text {stat }}$ has been found to give the best performance in separating photon-induced air shower events from the background of hadron-induced events. To take into account the energy and zenith angle dependences of the three observables, also energy and zenith angle are included in the BDT. Overall, the background contamination at a photon selection efficiency of $50 \%$ is $0.14 \%$ under the worst-case assumption of a pure proton background. The impact of different assumptions on the background contamination has been studied, e.g. by changing the hadronic interaction model (the background contamination changes from $0.14 \%$ to $0.21 \%$ ) or by using a mixture of $50 \%$ proton and $50 \%$ iron as background (the background contamination decreases to $0.04 \%$ ). To identify photons, a candidate cut is defined at the median of the BDT response distribution for primary photons. This way, the signal efficiency remains constant independently of any assumptions on the composition and the hadronic interactions.
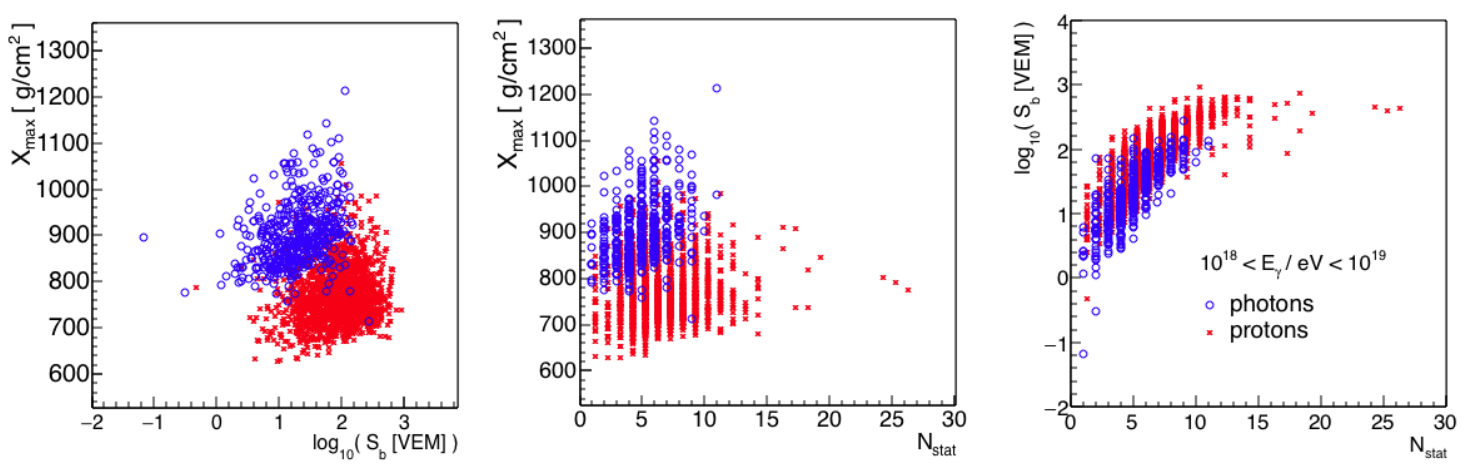

Figure 1: Correlation between the discriminating observables used in the MVA for the energy range $10^{18}<$ $E_{\gamma}<10^{19} \mathrm{eV}$ [5]: the simulated photon sample is shown as blue circles, while the proton sample is denoted by red stars. 

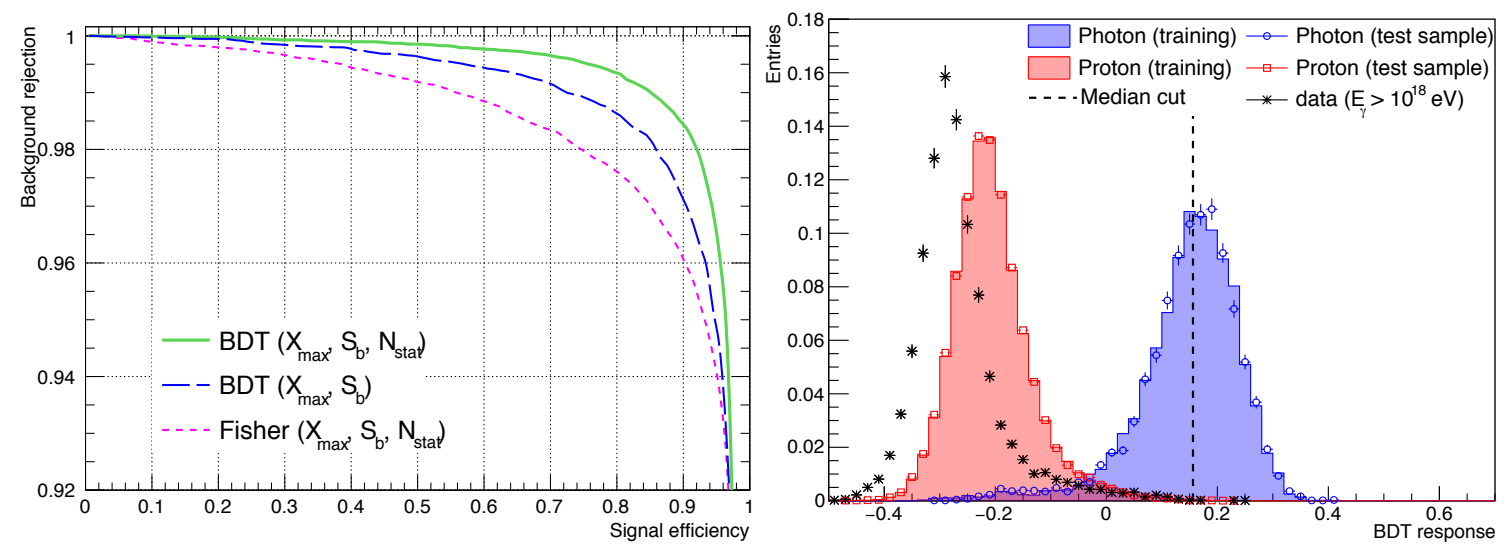

Figure 2: Left: background rejection efficiency as a function of the signal efficiency for different MVA algorithms; right: distribution of the BDT response for the signal sample (photon simulations, blue), background sample (proton simulations, red) and data (black); the photon candidate cut at the median of the photon distribution is indicated by the dashed line [5].

The analysis is then applied to hybrid data collected between January 2005 and December 2013. Selection criteria are applied to ensure a good geometry and profile reconstruction and a reliable determination of the discriminating observables. After the event selection, 8178 events remain for the analysis with energies above $10^{18} \mathrm{eV}$. The reference energy used here is the photon energy $E_{\gamma}$, i.e. the calorimetric energy determined from the longitudinal profile with a missing energy correction of $1 \%$ suitable for photon-induced air showers applied. The BDT response is given in Fig. 2, right, for the data and the simulated photon and proton samples for comparison. The discrepancy between the data and the proton simulations is in agreement with current experimental indications of a change to a heavier composition in the EeV range [8] and the muon deficit observed in simulations with respect to Auger data [9]. Three events pass the photon candidate cut, with 11.4 (3.3) expected for a pure-proton (mixed) background.

Since the number of selected photon candidates is compatible with the background expectation, upper limits (UL) on the integral photon flux at 95\% confidence level (C.L.) are derived as:

$$
\Phi_{\mathrm{UL}}^{0.95}\left(E_{\gamma}>E_{0}\right)=\frac{N_{\gamma}^{0.95}\left(E_{\gamma}>E_{0}\right)}{\mathscr{E}_{\gamma}\left(E_{\gamma}>E_{0} \mid E_{\gamma}^{-\Gamma}\right)},
$$

where $N_{\gamma}^{0.95}$ is the Feldman-Cousins upper limit at $95 \%$ C.L. on the number of photon candidates assuming zero background events and $\mathscr{E}_{\gamma}$ is the integrated exposure, derived from MC simulations, above the energy threshold $E_{0}$, under the assumption of a power law spectrum $E^{-\Gamma}$ with $\Gamma=2$. Upper limits to the integral photon flux are set to $0.027,0.009,0.008,0.008,0.007 \mathrm{~km}^{-2} \mathrm{sr}^{-1} \mathrm{yr}^{-1}$ for energy thresholds of 1, 2, 3, 5 and $10 \mathrm{EeV}$. The three candidate events all have energies close to $1 \mathrm{EeV}$, so the observed number is zero for all but the first energy threshold. The upper limit for $E_{0}=1 \mathrm{EeV}$ has been derived under the conservative choice that the three candidate events are indeed induced by photons, as if there were no expected background. This makes the limits more robust against hadronic interaction and mass composition assumptions. Rescaling the photon flux limits by the measured all-particle spectrum [10] results in photon fraction limits of $0.1 \%, 0.15 \%$, $0.33 \% 0.85 \%$ and $2.7 \%$ for the same energy thresholds. 


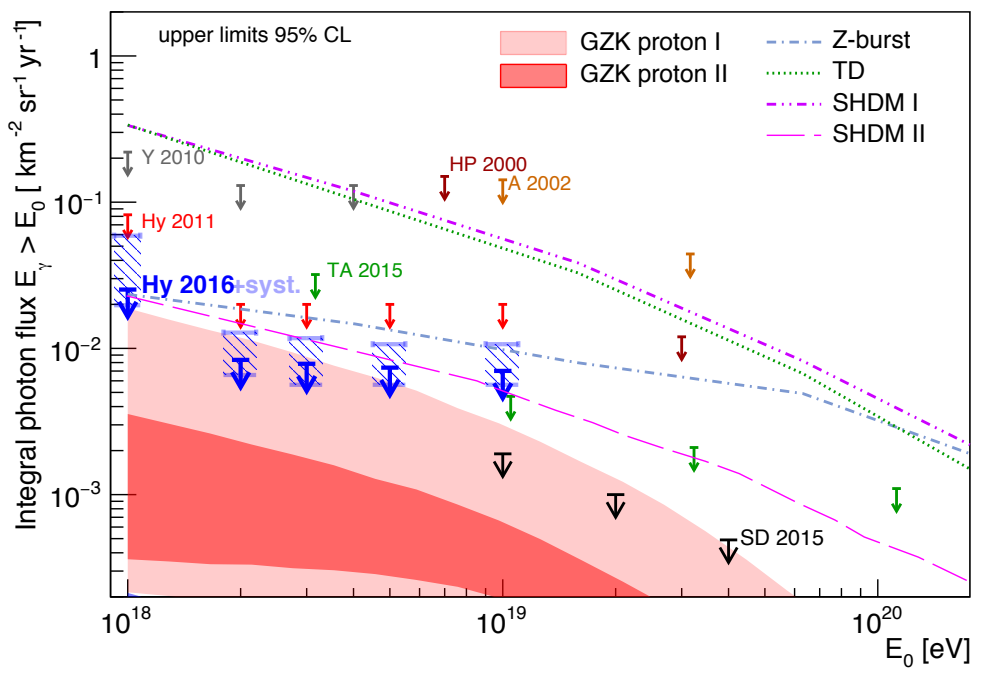

Figure 3: Upper limits on the integral photon flux derived in the analysis presented here (blue arrows, Hy 2016) [5]. The limits obtained when the detector systematic uncertainties are taken into account are indicated by the light-blue dashed boxes around the blue arrows. Also shown are the limits previously published by the Pierre Auger Observatory (Hy 2011 and SD 2015) and other experiments (Telescope Array, Yakutsk, AGASA, Haverah Park). The shaded regions and the lines give the predictions for photon fluxes from GZKbased models and several top-down models (Z-burst, topological defects, super-heavy dark matter). For a full list of references, see [5].

The upper limits derived in the analysis presented here are shown in Fig. 3 in comparison to previous results and predictions from several theoretical models. The new results lower the upper limits on the photon fraction by up to a factor 4 compared to previously published limits (Hybrid 2011 in Fig. 3), due to the larger dataset used in this analysis and the improvements in the background rejection. The robustness of the results has been tested against several sources of systematic uncertainties, for example uncertainties on the energy scale, or uncertainties in the determination of the discriminating observables. The impact of the systematic uncertainties on the upper limits are indicated in Fig. 3. The current upper limits impose tight constraints on current top-down scenarios proposed to explain the origin of UHE cosmic rays. The achieved sensitivity allows testing photon fractions of about $0.1 \%$ and exploring the region of photon fluxes predicted in some astrophysical scenarios (indicated by the shaded areas in Fig. 3).

\section{Targeted search for point sources of photons}

In the analysis described in the previous section, the search for UHE photons has been performed without using the reconstructed arrival direction of the recorded air shower events. Since photons, unlike charged cosmic rays, are not deflected by galactic and extragalactic magnetic fields, they point back to their production site. Since the production mechanisms for UHE photons are closely tied to UHE cosmic rays, a search for point sources of UHE photons could also help to pinpoint the sources of UHE cosmic rays. In the data, a photon point source would be detectable through an excess of photon-like events from a certain direction in the observed sky. Previously, the Pierre Auger Collaboration published a blind search for photon point sources [11]. No evidence 
for an excess of photon-like events has been found for any direction in the sky. The targeted search discussed in the following section complements the blind search by restricting the analysis to predefined target classes to reduce the statistical penalty of many trials. Since the attenuation length of photons in the energy range considered here $\left(10^{17.3}\right.$ to $\left.10^{18.5} \mathrm{eV}\right)$ varies between 90 and $900 \mathrm{kpc}$ [6], these target classes contain mostly galactic sources such as, e.g., millisecond pulsars, $\gamma$-ray pulsars, and low-mass and high-mass X-ray binaries as well as the Galactic center. In addition, two nearby extragalactic target sets are included: three powerful $\gamma$-ray emitters in the Large Magellanic Cloud and the core region of Centaurus A. The different target classes are listed in Tab. 1. A more detailed description can be found in [6]. The analysis uses hybrid events from the same data period as in the search for a diffuse flux of photons (January 2005 to December 2013), but in a different energy range $\left(10^{17.3}\right.$ to $\left.10^{18.5} \mathrm{eV}\right)$ to take advantage of the higher statistics at lower energies. In total, 308,676 well-reconstructed events enter the analysis. The average angular resolution of this data set is $0.7^{\circ}$.

To reduce the contamination of hadronic background events, photon-like air showers are selected using a BDT trained with MC simulations of photon- and proton-induced air shower events. The main input observables of the BDT are $X_{\max }$ and $S_{b}$, similar to the search for a diffuse flux of photons described in the preceding section. These two observables are complemented by additional observables [6]: the reduced $\chi^{2}$ of the fit of a Greisen function to the recorded longitudinal profile, the normalized energy derived from the Greisen function, and the ratio of the early-arriving to the late-arriving signal in the surface detector with the highest signal. Photon-like events are then selected through a cut in the BDT response $\beta$. This cut is optimized for each target direction by taking into account the expected number of background events, which has been derived using the scrambling technique [12]. Averaged over all target directions, the photon selection cut is expected to retain $81.4 \%$ of primary photons with a background rejection of $95.2 \%$. To determine how many events arrive from a given target direction in the data sample, a top-hat counting region

\begin{tabular}{l|ccc||ccc}
\hline \hline Class & $N$ & $\mathscr{P}$ & $\mathscr{P}_{w}$ & $p$ & $p^{*}$ & $f_{\mathrm{UL}}^{0.95}\left[\mathrm{~km}^{-2} \mathrm{yr}^{-1}\right]$ \\
\hline msec PSRs & 67 & 0.14 & 0.57 & 0.010 & 0.476 & 0.043 \\
$\gamma$-ray PSRs & 75 & 0.98 & 0.97 & 0.007 & 0.431 & 0.045 \\
LMXB & 87 & 0.74 & 0.13 & 0.014 & 0.718 & 0.046 \\
HMXB & 48 & 0.84 & 0.33 & 0.040 & 0.856 & 0.036 \\
H.E.S.S. PWN & 17 & 0.90 & 0.92 & 0.104 & 0.845 & 0.038 \\
H.E.S.S. other & 16 & 0.52 & 0.12 & 0.042 & 0.493 & 0.040 \\
H.E.S.S. UNID & 20 & 0.45 & 0.79 & 0.014 & 0.251 & 0.045 \\
Microquasars & 13 & 0.48 & 0.29 & 0.037 & 0.391 & 0.045 \\
Magnetars & 16 & 0.89 & 0.30 & 0.115 & 0.858 & 0.031 \\
Gal. Center & 1 & 0.59 & 0.59 & 0.471 & 0.471 & 0.024 \\
LMC & 3 & 0.62 & 0.52 & 0.463 & 0.845 & 0.030 \\
Cen A & 1 & 0.31 & 0.31 & 0.221 & 0.221 & 0.031 \\
\hline \hline
\end{tabular}

Table 1: Combined unweighted probabilities $\mathscr{P}$ and weighted Probabilities $\mathscr{P}_{w}$ for the 12 target sets [6]. In addition, selected information on the most significant target from each target set is given: the unpenalized $(p)$ and penalized $\left(p^{*}\right) p$-values and the upper limit on the photon flux at $95 \%$ C.L.. More details on the most significant targets, e.g. the galactic coordinates and upper limits on the energy flux from this target, can be found in [6]. 
of $1^{\circ}$ is used. After applying the photon selection cut, the total number of events from all target directions is 474.

A $p$-value $p_{i}$ is assigned to each candidate source $i$ of a target set, taking into account the observed number of events from this target direction as well as the expected number of background events. The $p$-values of all targets in a set are combined with and without statistical weights $w_{i}$. The weight assigned to each target is proportional to both the measured electromagnetic flux from the source (taken from astrophysical catalogs) and the directional exposure for photons, derived from simulations. The combined weighted probability $\mathscr{P}_{w}$ is the fraction of isotropic simulations yielding a weighted product $\prod_{i} p_{i \text {,iso }}^{w_{i}}$ that is not greater than the measured weighted product $\prod_{i} p_{i}^{w_{i}}$ :

$$
\mathscr{P}_{w}=\operatorname{Prob}\left(\prod_{i} p_{i, \text { iso }}^{w_{i}} \leq \prod_{i} p_{i}^{w_{i}}\right),
$$

where $p_{i, \text { iso }}$ denotes the $p$-value of target $i$ in an isotropic simulation. The combined unweighted probability $\mathscr{P}$ is given by the same formula with $w_{i}=1$ for all targets. The results of the analysis for each of the 12 target sets are shown in Tab. 1, along with information about the target with the smallest $p$-value in each set. In addition, the penalized $p$-values $p^{*}=1-(1-p)^{N}$ are given, which is the chance probability that one or more of the $N$ targets in the set have a $p$-value less than $p$ under the assumption of a uniform probability distribution. No combined $p$-value (weighted and unweighted) nor any individual $p$-value for a target has a statistical significance as great as $3 \sigma$. No target class therefore reveals compelling evidence for photon-emitting sources in the $\mathrm{EeV}$ range. There is also no evidence for one outstanding target in any target set.

Upper limits on the photon flux from the targets with the smallest $p$-values in the sets are calculated according to

$$
f_{\mathrm{UL}}^{0.95}=\frac{n_{\mathrm{Zech}}^{0.95}}{\varepsilon \times n_{\mathrm{inc}}},
$$

where $n^{\text {Zech }}$ is the upper limit, at $95 \%$ C.L., on the number of photons obtained using Zech's method [13], $\varepsilon$ is the directional exposure to photons and $n_{\text {inc }}$ is the expected signal fraction within the search window. The resulting upper limits for the individual targets are listed in Tab. 1 as well.

Of particular interest is the limit on the Galactic center. The H.E.S.S. collaboration recently reported evidence for the acceleration of $\mathrm{PeV}$ protons in this region [14]. Fig. 4 shows the spectrum measured by H.E.S.S. in the $\mathrm{TeV}$ range, along with an extrapolation of this spectrum to $\mathrm{EeV}$ energies. This extrapolation also takes into account interactions with, for example, the CMB. The flux limit derived in the analysis presented here can severely constrain the allowed parameter space for such an extrapolation. Furthermore, assuming a power law with an exponential cutoff, an upper limit on the cutoff energy of $2 \mathrm{EeV}$ can be placed. The corresponding spectrum is also shown in Fig. 4.

\section{Summary}

So far, the extensive searches for UHE photons at the Pierre Auger Observatory have not yielded an unambiguous detection of photons at these energies. The upper limits on the diffuse flux of photons presented here are the most stringent limits to date, severely constraining top-down 


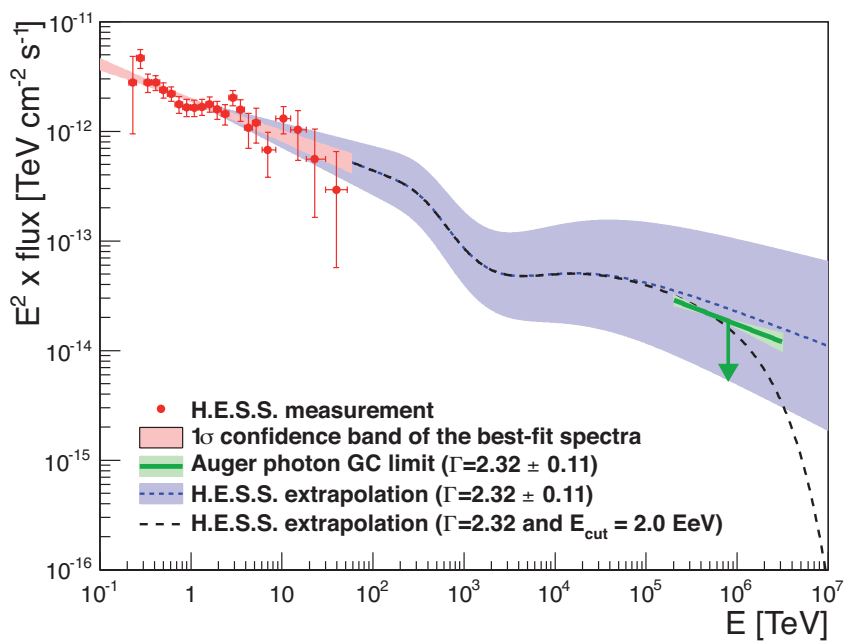

Figure 4: Photon flux as a function of energy from the Galactic center region [6]. The flux measured by H.E.S.S. in the TeV range [14] is shown in red, as well as the extrapolation to the EeV range (blue dashed line). The upper limit on the flux derived in the analysis presented here is given in green. A spectrum with an exponential cutoff at $2 \mathrm{EeV}$ is shown as the black dashed line.

models for the origin of UHE cosmic rays. The targeted search for photon point sources likewise yielded no evidence for EeV photon emitters in any of the studied source classes. However, the connection to measurements from the $\mathrm{TeV}$ range enables new multi-messenger studies, for example in studies of the Galactic center.

\section{References}

[1] F. Aharonian, et al., Astron. Astrophys. 464 (2007) 235.

[2] The Pierre Auger Collaboration, Phys. Rev. Lett. 101 (2008) 061101.

[3] M. Risse and P. Homola, Mod. Phys. Lett. A 22 (2007) 749.

[4] The Pierre Auger Collaboration, Nucl. Instr. Meth. Phys. Res. A 798 (2015) 172.

[5] The Pierre Auger Collaboration, J. Cosmol. Astropart. Phys. 04 (2017) 009.

[6] The Pierre Auger Collaboration, Astrophys. J. 837 (2017) L25.

[7] G. Ros, et al., Astropart. Phys. 35 (2011) 140.

[8] The Pierre Auger Collaboration, Phys. Rev. D 90 (2014) 122005.

[9] The Pierre Auger Collaboration, Phys. Rev. D 91 (2015) 032003.

[10] I. Valiño for the Pierre Auger Collaboration, PoS(ICRC2015) 271.

[11] The Pierre Auger Collaboration, Astrophys. J. 789 (2014) 160.

[12] G. L. Cassiday, et al., Nucl. Phys. Proc. Suppl. 14A (1990) 291.

[13] G. Zech, Nucl. Instr. Meth. Phys. Res. A 277 (1989) 608.

[14] The H.E.S.S. Collaboration, Nature 531 (2016) 476. 